\title{
Nuove prospettive francesi sul Rinascimento italiano
}

\section{Letizia Norci Cagiano}

\section{(2) OpenEdition}

1 Journals

\section{Edizione digitale}

URL: http://journals.openedition.org/studifrancesi/7442

DOI: $10.4000 /$ studifrancesi.7442

ISSN: 2421-5856

\section{Editore}

Rosenberg \& Sellier

\section{Edizione cartacea}

Data di pubblicazione: 1 décembre 2009

Paginazione: 557-562

ISSN: 0039-2944

\section{Notizia bibliografica digitale}

Letizia Norci Cagiano, «Nuove prospettive francesi sul Rinascimento italiano», Studi Francesi [Online], 159 (LIII | III) | 2009, online dal 30 novembre 2015, consultato il 08 janvier 2021. URL: http:// journals.openedition.org/studifrancesi/7442 ; DOI: https://doi.org/10.4000/studifrancesi.7442

\section{(c) (†) $\odot$}

Studi Francesi è distribuita con Licenza Creative Commons Attribuzione - Non commerciale - Non opere derivate 4.0 Internazionale. 


\section{DISCUSSIONI E COMUNICAZIONI}

\section{Nuove prospettive francesi sul Rinascimento italiano}

«L'histoire, en général, s’occupe du passé, scriveva Jacques Thuiller nel 1995. L'histoire littéraire et l'histoire de l'art s'occupent d'objets présents qui ont un passé»' e che, potremmo aggiungere, alla storia di quel passato hanno contribuito in modo determinante. Questa è la tesi di fondo di uno studio di Édouard Pommier, Comment l'art devient l'Art dans l'Italie de la Renaissance, uscito contemporaneamente in Francia (Gallimard, 2007) e in Italia, nella versione di Chiara Bongiovanni (L'invenzione dell'arte nell'Italia del Rinascimento, Einaudi, 2007). La diffusione simultanea dell'opera nei due paesi non desta meraviglia, dato che Édouard Pommier, nelle sue opere come nelle sue relazioni umane e scientifiche, è sempre stato ambasciatore di una concezione della cultura che non conosce frontiere nazionali.

Il suo studio riposa su vaste letture e su una conoscenza minuziosa dell'arte italiana: posizione privilegiata per un francese che voglia affrontare un tema impegnativo come quello in questione. Tuttavia, nell'intento di offrire un affresco inedito e problematico del Rinascimento italiano, Pommier si è imposto, come punto di partenza, di dimenticare tutta la letteratura sul Rinascimento e di non pensare che lui, francese, affrontava un tema visceralmente legato agli Italiani. Questo sforzo iniziale gli ha permesso di partire da prospettive originali per costruire un'opera di alto valore per gli storici dell'arte e per gli storici (Pommier dimostra come la storia d'Italia sia fatta in gran parte dagli artisti), ma anche per il lettore comune in virtù della chiarezza, della varietà dei toni, delle interessanti illustrazioni e della compiutezza di ciascun capitolo. Infatti, se il discorso si snoda con coerenza e con progressione anche cronologica nell'arco del volume, le singole parti possono essere lette come saggi conclusi e densi di informazioni e di suggestioni impreviste.

Il libro si fonda principalmente su quella metastoria dell'arte che sono le Vite del Vasari, pur nella consapevolezza, da parte dell'autore, dell'inattualità della visione ideale e provvidenzialistica di quell'opera che pure resta la fonte più ricca sulla storia della pittura italiana fino all'inizio del Cinquecento. E l'autore pensa, come Vasari, e contro chi sostiene che il Quattrocento italiano sia una specie di appendice del Medioevo, che un movimento nuovo si delinea in Toscana intorno al 1300, e che questo movimento non cessa di rafforzarsi e di svilupparsi in una continuità che giunge fino alla metà del XIX secolo. Sotto questo aspetto Pommier si ricollega ai grandi "inventori" francesi del Rinascimento, da Jules Michelet ad André Chastel, che fra l'altro hanno permesso a noi italiani di cogliere aspetti della nostra storia che ci sfuggono (quasi come i Persiani di Montesquieu nei confronti dei parigini del primo Settecento). Tuttavia l'impianto dell'opera di Pommier si distingue da quello dei suoi predecessori: non ci troviamo di fronte ad una trattazione sistematica, ma, come ho accennato, ad una sequenza di quadri, di sondaggi, di tessere che formano

(1) “RHLF”, 1995, n. 6, p. 151. 
un mosaico non convenzionale, finalizzato ad un'interpretazione della storia dell'arte italiana, dal Trecento a Michelangelo, che non segue i canoni tradizionali delle scuole o delle tendenze più rappresentative, ma osserva la progressiva conquista, da parte dell'artista, di un ruolo nella società che lo circonda.

Le Vite del Vasari si aprono su una processione trionfale che accompagna la Madonna Rucellai a Santa Maria Novella (1285 circa) e si concludono con un altro fastoso corteo, quello dei funerali di Michelangelo a San Lorenzo (1564): il percorso dal trionfo di un'opera d'arte (e non, si badi bene, di un'immagine sacra) al trionfo di un artista costituisce la trama del libro di Édouard Pommier. A partire dall'esaltazione di un dipinto, La Madonna Rucellai (che Vasari attribuisce erroneamente a Cimabue, ma che sappiamo essere opera di Duccio di Buoninsegna), in cui i contemporanei scorgono una novità se non addirittura una rottura con la pittura tradizionale, Pommier mostra come, attraverso i secoli seguenti, alla conquista di uno statuto e di una dignità da parte dell'artista, si associ l'invenzione di un insieme complesso di testi e di immagini, di monumenti e di istituzioni (vite di artisti, ritratti, elogi, accademie) che costituiscono un vero e proprio cortège: questo invade i margini del fatto artistico propriamente detto, dotandolo di una specie di valore aggiunto che entra a far parte della sua specificità e lo rende protagonista della storia.

Secondo Vincenzo Borghini, contemporaneo e amico di Vasari, meritano di essere scritte soltanto le vite dei Principi, e non quelle di persone di bassa condizione, come gli artisti, che nulla hanno a che fare con la storia. Questo criterio, ormai inattuale quando Borghini scrive, era invece generalmente adottato all'epoca di Giotto: vuoi nel De viris illustribus di Petrarca, dove non si trovano artisti tra coloro che hanno reso illustre la patria, vuoi nelle sculture per il Campanile di Firenze eseguite da Andrea Pisano e dai suoi allievi. Qui, nei bassorilievi del basamento, la pittura e la scultura non sono rappresentate attraverso allegorie o nelle vesti di donne belle e maestose come avveniva per le arti liberali, ma come mestieri eseguiti da artigiani.

È comunque al tempo di Giotto, e soprattutto grazie a Giotto, che l'artista comincia ad assumere un nuovo ruolo; già Dante, nell'XI canto del Purgatorio parla di gloria a proposito di Cimabue e del suo celebre allievo; poco più tardi Boccaccio, nel Decamerone, associa quell'eccelso pittore alla grandezza di Firenze: Giotto merita la gloria perché è un uomo illustre che contribuisce alla storia della sua città.

Dall'intuizione di Boccaccio emerge l'aspetto politico dell'invenzione di una storia dell'arte legata alla coscienza collettiva di una città che fa dell'integrazione della pittura alle sue vicende un mito fondatore della sua identità. Ma il ruolo del grande artista non si ferma qui, perché Giotto viaggia e dipinge in diverse città d'Italia contribuendo a illustrare oltralpe un'immagine collettiva del Paese attraverso la sua opera e la sua personalità; è d'altronde quello che aveva fatto Petrarca nel campo delle lettere. La visione dell'Italia che viene esportata in Francia e che tanto contribuirà alla configurazione della Renaissance, non sarà dunque quella frammentata di una somma di stati e staterelli, ma quella d'insieme fornita dalla Repubblica delle lettere e, ormai, delle arti.

L'affermazione dell'artista nel ruolo di rappresentante illustre o anche di protagonista della storia si compie attraverso un cammino complesso che Pommier segue in tutti i suoi détours, con un dispiegamento di esempi che rivelano una mole straordinaria di letture e di conoscenze: lo sguardo, pur essendo essenzialmente toscanocentrico, si sposta da Firenze a Roma e dalle grandi personalità di artisti a quelle Accademie delle arti il cui modello, nel Seicento, s'irradierà dall'Italia in tutta Europa, così come dall'Italia s'irradierà, un secolo più tardi, il concetto e la pratica del Museo.

Il Museo introduce al problema della tutela, oggi di grande attualità e di portata internazionale: di recente è stata inaugurata al Colosseo una mostra, Rovine e rinascite dell'arte in Italia (catalogo, Milano, Electa, 2008) che ripercorre le vicende 
di leggi, trasgressioni, fughe e ritrovamenti di opere d'arte a partire dall'Unità fino ai giorni nostri. Pommier parte invece dal Trecento, quando l'identità dell'Italia si basava unicamente sul suo patrimonio culturale e gli artisti inventarono l'immagine dei "monumenti", intesi come testimonianze figurate della civiltà greco-romana, quindi memoria di una gloriosa eredità comune. In quest'ottica a Roma, fin dal Quattrocento, si agisce per la salvaguardia di opere ereditate dal passato (Sisto IV nel 1471 tutela alcuni grandi capolavori dell'antichità, "restituendoli" al popolo romano), si delinea la nozione di patrimonio e si pongono le basi per l'istituzione del Museo che nascerà, qualche secolo più tardi, come luogo di custodia, di gestione e di promozione del patrimonio romano.

La questione della tutela e della promozione costituisce solo uno degli aspetti del libro di Pommier, e forse neppure il più importante, ma da qui vorrei prendere spunto per alcune brevi considerazioni che riguardano gli esiti sei-settecenteschi di quell'opera di trasmissione della politica artistica italiana in Francia che era culminata all'epoca di François $1^{\text {er }}$.

Agli artisti italiani del Rinascimento spettò, come abbiamo accennato, una missione di recupero e di valorizzazione dei "monumenti", che si concretizzò attraverso l'imitazione dei modelli antichi (pensiamo all'influsso dei sarcofaghi romani sulle sculture di Nicola e Giovanni Pisano), l'impegno nel restauro e nell'allestimento delle collezioni, ma anche nella pratica, diffusa a partire dalla metà del Quattrocento, d'inserire nei dipinti immagini di sculture e di architetture antiche. Gli esempi proposti da Pommier, che riguardano pittori operanti in varie parti d'Italia, possono suggerire inediti itinerari artistici. A Roma, per esempio, potrebbe essere facile e interessante passare dal monumento originale alla sua rappresentazione: dal Marco Aurelio, nel suo nuovo allestimento all'interno dei Musei Capitolini, all'immagine che Filippino Lippi ne ritrae sullo sfondo dell'affresco della cappella Carafa a Santa Maria sopra Minerva; dall'Arco di Costantino nel complesso monumentale del Colosseo, alla ricostruzione che ne fa Botticelli nella scena della Punizione di Core, Dathan e Abiron alla Cappella Sistina.

Questa pratica, come è noto, fu ripresa dagli artisti francesi che soggiornarono a Roma, primo fra tutti Poussin che molto si esercitò sui modelli antichi e che spesso utilizzava come sfondo per i suoi quadri architetture romane ispirate a Vitruvio o ai suoi seguaci cinquecenteschi. Come avviene per Botticelli nell'affresco della Sistina dove l'Arco di Costantino domina in una scena biblica, per Poussin non aveva importanza che l'azione si svolgesse a Roma, in Grecia, o nei luoghi delle Sacre Scritture; le sue ricostruzioni architettoniche, archeologicamente inesatte, volevano dare un'immagine di quell'Antichità la cui perfetta magnificenza si poneva come modello incontestabile per la Francia del XVII secolo ${ }^{2}$. Un'operazione analoga compie Corneille mettendo in scena eroi che si ispirano ad una grandeur romana ideale e si muovono in ambienti ricostruiti secondo una visione acritica del mondo antico; procedimento ben messo in evidenza da Guez de Balzac nel suo elogio di Cinna:

Vous nous faittes voir Rome tout ce qu'elle peut être à Paris, et ne l'avez point brisée en la remuant. Ce n'est point une Rome de Cassiodore, et aussi déchirée qu'elle était au siècle des Théodorics: c'est une Rome de Tite-Live, et aussi pompeuse qu'elle était au temps des premiers Césars [...] Aux endroits où Rome est de brique, vous la rebâtissez de marbre; quand vous trouvez du vide, vous le remplissez d'un chef-d'œuvre, et je prends garde que ce que

(2) Cf. a questo proposito l'illuminante articolo di C. L. Frommel, Poussin e l'architettura, in Poussin et Rome, Actes du colloque à l'Académie de
France à Rome et à la Biblioteca Hertziana, s.e., Paris,1996, pp. 119-134. 
vous prêtez à l'histoire est toujours meilleur que ce que vous empruntez d'elle (lettera del 17 gennaio 1643).

Questi esempi di appropriazione e di riconversione di una pratica italiana in ambito artistico e letterario sono contemporanei alla creazione in Francia di Accademie delle Arti che, pur partendo da un modello d'oltralpe che andava diffondendosi in tutt'Europa, assumono caratteri e finalità assolutamente originali nel corso del Seicento.

Si dovrà invece attendere il XVIII secolo per riscontrare in Francia gli esiti della politica di conservazione e valorizzazione dei "monumenti" intrapresa da Papi e Principi del Rinascimento. Proprio verso la fine del Seicento si può notare che, tra le principali ragioni del viaggio in Italia, emerge la ricerca di testimonianze concrete della storia antica: iscrizioni, gemme, statue, mosaici, pitture, resti di edifici che sono stati miracolosamente preservati. Malgrado la decadenza, l'esodo e la dispersione di tante opere, l'Italia resta una miniera di vestigia accessibili che offrono oltre a una maggiore o minore fruizione estetica, la possibilità di verificare scientificamente i racconti degli Autori, secondo le esigenze che erano emerse dalla "Querelle des Anciens et des Modernes".

Nei primi decenni del Settecento i Francesi restano dunque ammirati dalle collezioni di antichità che vengono liberalmente aperte ai visitatori a Venezia, Verona, Firenze, Roma e s'interessano alle opere di studio, di catalogazione, di restauro e di esposizione dei reperti, che in quegli anni si andavano avviando. Montesquieu, nel 1728 , percorre in lungo e in largo il Palatino, senza trascurare l'iscrizione di Francesco Bianchini che riporta gli esiti delle sue recenti indagini sulle proporzioni, i materiali e le tecniche impiegate per la costruzione del palazzo3.

Dieci anni più tardi il presidente de Brosses avrà modo di visitare le raccolte di antichità che i papi accumulavano in Campidoglio. Soprattutto dal tempo di Clemente XI e in seguito con l'apporto delle collezioni Albani acquistate da Clemente XII, le sale traboccavano di sculture:

Cela est répandu presque sans ordre dans les cours des ailes, soubs les portiques, sur les escaliers, dans les apartemens. Il seroit bien temps que l'on y mit un bel arrangement, dont ce recueil seroit susceptible et alors cette galerie ne seroit nullement inférieure à celle du grandduc [cioè la Galleria degli Uffizi che Clemente XII Corsini ben conosceva]; mais je crois que l'espace leur manque. On paroît néamoins dans le dessein d'y travailler bientost, d'y joindre en même temps tout ce que l'on poura acquérir dans Rome et d'y former même un recueil de tableaux. Ceci dépend de sçavoir si le pape qui viendra aprez celuy-ci sera un homme de goût et s'il trouvera de l'argent pour suffire à la dépense ${ }^{4}$.

Per fortuna il cardinale Lambertini era tra i sostenitori di quella strategia per la promozione di Roma attraverso la valorizzazione delle sue antichità e dei suoi tesori artistici; così, quando nel 1740 salì al soglio pontificio, incoraggiò la creazione della Pinacoteca capitolina e la riorganizzazione dei Musei, affidando a Giovanni Bottari la cura dei cataloghi di antichità .

La pubblicazione dei cataloghi rientrava da tempo nelle abitudini degli antiquari italiani: gli stranieri che non potevano accedere di persona alle raccolte, o che volessero ripercorrere con la memoria le cose viste, potevano utilizzare splendidi volumi

(3) Gli studi di Bianchini saranno pubblicati postumi nel 1738 (Il Palazzo dei Cesari, Verona, Berno).

(4) Charles de Brosses, Lettres familières, edizione critica, Napoli, Centre Jean Bérard, 3 vol.,
1991, II, p. 863.

(5) Giovanni Bottari, Del Museo Capitolino, Roma, 3 volumi pubblicati da diversi editori, 17411755 . 
illustrati come quello degli Zanetti, Delle antiche statue greche e romane che nell'antisala della Libreria di San Marco, e in altri luoghi pubblici di Venezia si trovano ${ }^{6}$ o l'imponente raccolta d'incisioni in 10 volumi uscita a Firenze presso Michel Nestemus e Francesco Moücke dal 1731 al 1762 con le immagini di gemme, statue, medaglie ed altre opere d'arte custodite agli Uffizi e in qualche collezione privata della città. Queste opere, e molte altre dello stesso genere, cui bisogna aggiungere gl'innumerevoli disegni dei pensionnaires dell'Accademia di Francia a Roma, fornivano quelle immagini dell'antichità che nutrirono in Francia la rinascita del "grand-goût" e, più tardi, i dibattiti intorno alle teorie di Winckelmann. I cataloghi illustrati italiani si proposero anche come modelli per imprese analoghe, come il Recueil d'antiquités di Caylus $^{7}$, rassegna vasta e minuziosa che troverà sviluppi straordinari nei "voyages pittoresques" che univano la descrizione di un itinerario di viaggio alla rappresentazione dei monumenti nel loro contesto storico e naturale.

Vivant Denon, protagonista e redattore del celebre Voyage Pittoresque ou Description des Royaumes de Naples et de Sicile di Saint-Non', è tra coloro che meglio seppero mettere a frutto l'esempio del Museo aperto al pubblico (anche se con qualche restrizione) offerto dai Papi e da molti signori italiani. Riallacciandosi al dibattito sul Museo già vivo in Francia prima della Rivoluzione (ed esaurientemente illustrato proprio da Édouard Pommier nel suo volume L'Art de la liberté), Denon sarà tra i fondatori e il primo direttore del Musée Napoléon, nato sotto la doppia costellazione dell'Italia e della Francia: non solo perché ricco di antichità e di opere d'arte provenienti dalla Penisola; non solo perché il direttore per le antichità fu Ennio Quirino Visconti, con tutto il suo bagaglio di esperienza nella catalogazione del Museo PioClementino; ma anche e soprattutto perché ispirato alla politica culturale dei Papi nell'ottica di uno scambio di ruoli epocale: la Parigi di Napoleone il grande è destinata a divenire la "nouvelle Rome".

Denon introduce grandi innovazioni nella concezione del Museo: in particolare la priorità data ad opere di altissima qualità artistica e alla possibilità di collocarle secondo una progressione cronologica coerente. Si tratta di una visione rivoluzionaria che tuttavia aveva potuto prender forma durante i soggiorni italiani di Denon: a Roma, dove i monumenti antichi si fondono senza soluzione di continuità con le architetture medioevali, rinascimentali, barocche; o a Firenze dove da Giotto a Raffaello una moltitudine di pittori allora non abbastanza considerati segna le tappe di un'arte che si sviluppa in concomitanza con la storia e che della storia è partecipe e talvolta protagonista. Ecco dunque che nelle splendide sale del Louvre i pittori-artigiani, i cosidetti "primitivi" riacquistano d'emblée quella importanza che il Classicismo aveva offuscato.

Molti di questi dipinti non restano a Parigi per più di vent'anni; è quel tanto che basta perché al loro ritorno nelle antiche sedi, in seguito ai decreti del Congresso di Vienna, siano investiti di un nuovo ruolo. Sull'esempio del Louvre i musei italiani impareranno a tener conto, nei limiti del possibile, di alcuni princìpi instaurati da Vivant Denon e ormai accettati in Europa: $i$ quadri tornati a Roma non saranno restituiti alle

(6) Venezia, s.e., 2 voll., 1740-1743.

(7) Anne Claude Philippe de Caylus, Recueil d'Antiquités égyptiennes, étrusques, grecques et romaines, Paris, Desaint et Saillant, 6 voll., 17521767.

(8) Jean Claude Richard de Saint-Non, Voyage Pittoresque ou Description des Royaumes de Naples et de Sicile, Paris, Clousier, 5 voll., 1781-1786. E no- to che, all'uscita di quest'opera, Denon si dissociò dall'impresa e pubblicò il suo diario della spedizione in Sicilia e a Malta in appendice alla traduzione francese del viaggio di Swinburne. Il racconto di Denon costituisce infatti il V tomo del Voyage de Henri Swinburne dans les Deux Siciles, Paris, Didot l'aîné, 1787. 
chiese o ai monasteri di provenienza, ma verranno esposti in collezioni pubbliche; inoltre si provvederà, sotto il pontificato di Gregorio XVI, alla raccolta dei dipinti di bizantini e di "primitivi". In generale, dalla metà dell'Ottocento in poi, gli allestimenti dei musei romani (compresi i nuovi settori dei Vaticani) terranno conto dei criteri storicistici e didascalici adottati per la prima volta al Louvre.

Sull'opportunità di questi criteri il dibattito oggi è molto vivace; non si può tuttavia ignorare il valore aggiunto dell'operazione di Denon, partita da uno strettissimo rapporto tra Italia e Francia. Resta poi il fatto che il primo direttore del Louvre, attraverso l'esposizione simultanea e ordinata di tutti gli elementi di un'evoluzione artistica, ha permesso di abbracciare con lo sguardo, in un unico luogo, quel lungo e difficile percorso che Pommier descrive, tappa per tappa, nel suo libro. 